\title{
Clonal origin of aminoglycoside-resistant Citrobacter freundii isolates in a Danish county
}

\author{
N. NØRSKOV-LAURITSEN*†\#, D. SANDVANG*, J. HEDEGAARD§, V. FUSSING\|, \\ K. K. MORTENSEN\&, H. U. SPERLING-PETERSEN§ and H. C. SCHØNHEYDER* \\ * Department of Clinical Microbiology, Aalborg Hospital, DK-9000 Aalborg, †Department of Clinical \\ Microbiology, Aarhus University Hospital, DK-8000 Aarhus C, \$Danish Veterinary Laboratory, DK-1345 Kbh V, \\ $\S$ Department of Molecular and Structural Biology, Aarhus University, DK-8000 Aarhus C, /IDepartment of \\ Gastrointestinal Infections and \#Clinical Microbiology, Statens Serum Institut, DK-2300 Copenhagen S, \\ Denmark
}

\begin{abstract}
During 1997, attention was drawn to an increased frequency of aminoglycoside-resistant Citrobacter freundii in a Danish county, when a total of 24 resistant $C$. freundii isolates was detected. In this study, 15 such isolates were typed by pulsed-field gel electrophoresis, riboprinting and partial sequencing of the gene encoding translation initiation factor 2. Fourteen of the $\mathbf{1 5}$ isolates were identical, as evaluated by their antibiograms and by all these typing methods. This epidemic strain harboured the aminoglycoside resistance genes $\operatorname{aac}(3)-I I$ and $\operatorname{ant}\left(3^{\prime \prime}\right)-I$, with the latter located in tandem with a dihydrofolate reductase gene in a class $I$ integron. The source of the strain remains unresolved. Representative isolates were obtained from various specimens from hospitals and general practice throughout the county, with no evidence of patient-to-patient transmission.
\end{abstract}

\section{Introduction}

A recommended and widely employed empirical treatment for community-acquired sepsis in Denmark consists of an aminoglycoside in combination with a $\beta$ lactam antibiotic (usually penicillin or ampicillin) plus metronidazole $[1,2]$. Crucial to this strategy is a low frequency of aminoglycoside resistance among Enterobacteriaceae, which cause almost $50 \%$ of the bacteraemic episodes [2].

Prospective surveillance of gentamicin-resistant enteric rods was initiated in the county of Northern Jutland, Denmark, in 1993. The most marked observation was a sudden increase of gentamicin-resistant isolates of Citrobacter freundii, from none in 1994 to 24 in 1997 [3]. C. freundii is a species of Enterobacteriaceae with some similarity to Escherichia coli and Salmonella spp., and DNA sequence analysis shows that Citrobacter, Escherichia and Salmonella form one distinct lineage within the Enterobacteriaceae [4]. Nevertheless, and in contrast to its relatives, C. freundii is rarely implicated in human diarrhoea and enteritis; rather, it is an opportunist pathogen that can cause a

Received 14 Aug. 2000; revised version accepted 8 Dec. 2000.

Corresponding author: Dr N. Nørskov-Lauritsen (e-mail: prof1@aas.nja.dk). broad range of infections, the most common being those of the urinary tract. The species has a chromosomal class $\mathrm{C}(\mathrm{AmpC}) \beta$-lactamase that makes treatment outcomes with most cephalosporins unpredictable, and that confers ampicillin resistance [5].

Few suspected outbreaks of infection caused by Citrobacter spp. have been reported. However, in a case of vertical transmission of infections with $C$. koseri, the common identity of two isolates was demonstrated by pulsed-field gel electrophoresis (PFGE) and ribotyping [6], and in a nosocomial cluster of infections with $C$. koseri with extended-spectrum $\beta$ lactamases, six of eight isolates belonged to the same epidemic strain, as evaluated by ribotyping and arbitrarily-primed PCR [7].

Because of the restricted knowledge on the epidemiology of infection with $C$. freundii, this study aimed to characterise a sample of isolates from the suspected outbreak in Northern Jutland by three DNA-based methods.

\section{Materials and methods}

Collection, identification and susceptibility testing

The surveillance, identification and susceptibility testing of gentamicin-resistant enteric rods were described 
previously [3]. The isolates were kept at $-80^{\circ} \mathrm{C}$ in broth supplemented with glycerol $10 \% \mathrm{v} / \mathrm{v}$. Species identification was initially performed with a commercial system (Crystal RS/E, Becton-Dickinson, USA) that does not differentiate between the more recently described species in the $C$. freundii complex. On retrieval for the present study, the isolates were further characterised with respect to fermentation of sucrose, dulcitol and melibiose, utilisation of malonate, decarboxylation of ornithine and production of indole [8]. Based on these supplementary tests, speciation as $C$. freundii was confirmed. The isolates expressed highlevel resistance to gentamicin, with MICs $>256 \mathrm{mg} / \mathrm{L}$ as determined with E-tests (AB-Biodisk, Solna, Sweden).

\section{Macro-restriction fragment analysis by PFGE}

PFGE was done according to a protocol designed for investigations of $E$. coli $\mathrm{O} 157: \mathrm{H} 7$ [9]. Total cellular DNA was digested with the restriction endonucleases Spe I or XbaI (Pharmacia Biotechnology, Uppsala, Sweden). Electrophoresis was run on a CHEF-DRII apparatus (BioRad, Richmond, CA, USA) at $6 \mathrm{~V} / \mathrm{cm}$ for $22 \mathrm{~h}$ with an initial switch time of $1 \mathrm{~s}$ and a final switch time of $40 \mathrm{~s}$.

\section{Automated ribotyping}

Riboprinting was performed with the RiboPrinter ${ }^{\mathrm{TM}}$, used as recommended by the manufacturer (Qualicon, Wilmington, DE, USA). Briefly, single colonies from a 24-h culture on blood (5\%) agar were suspended in sample buffer and incubated at $80^{\circ} \mathrm{C}$ for $15 \mathrm{~min}$. After addition of lysis solution, the samples were transferred to the RiboPrinter ${ }^{\mathrm{TM}}$. Further analysis, including EcoRI restriction of DNA, was performed automatically. The riboprint profiles were aligned according to the position of a molecular size standard.

\section{infB sequencing}

PCR amplification and partial sequencing of $\inf B$, the gene encoding the prokaryotic translation initiation factor IF2, were performed as described previously [4]. A 417-nucleotide segment was selected in all sequences and was used for analysis. This fragment encodes the amino acid positions 424-562 with reference to E. coli IF2-1 [10].

\section{Amplification of resistance genes}

Aminoglycoside resistance genes (nomenclature according to Shaw et al. [11]) aac(3)-II, aac(3)-IV, $\operatorname{aac}\left(6^{\prime}\right)-I$, ant $\left(2^{\prime \prime}\right)-I$, ant $\left(3^{\prime \prime}\right)-I$, aph $\left(3^{\prime}\right)-I$ and $\operatorname{aph}\left(3^{\prime}\right)-$ II were amplified by PCR. Primers were chosen from the following sequences (GenBank accession numbers and position of nucleotides): X51534, 381-400 and 731-750; X01385, 311-330 and 919-938; X60321, 243-263 and 508-527; X04555, 1491-1510 and
1800-1819; D43625, 1407-1426 and 1916-1933; J01839, 1182-1202 and 1827-1851; and V00618, 200-222 and 725-746. A total of 28 amplification cycles was performed, each consisting of $94^{\circ} \mathrm{C}$ for $45 \mathrm{~s}$, $61^{\circ} \mathrm{C}\left(55^{\circ} \mathrm{C}\right.$ in the case of $\operatorname{aac}(3)-I I$ or $\left.\operatorname{aph}\left(3^{\prime}\right)-I I\right)$ for $45 \mathrm{~s}$ and $72^{\circ} \mathrm{C}$ for $1 \mathrm{~min}$. The amplification products were electrophoresed on agarose $1 \%$ gels and visualised under UV light after staining with ethidium bromide. PCR amplification of class 1 integrons was performed with primers Int1-F and qacE $\Delta 1-\mathrm{B}$, as described previously [12]. The amplification product of $c$. $2.2 \mathrm{~kb}$ from isolate ARC1 was purified with a QIAquick kit (Qiagen, Hilden, Germany) and subjected to sequencing with the same primers as used for the PCR. The initial sequencing revealed the presence of dhfrXII (encoding a dihydrofolate reductase conferring resistance to trimethoprim [13]) in the $5^{\prime}$-end of the amplification product, and $\operatorname{ant}\left(3^{\prime \prime}\right)-I$ in the $3^{\prime}$-end. Final sequencing of the entire PCR product was performed with the internal primers int1-B and ant(3')-I-B [12], AME 23-B [13] and dhfrXII-F [14]. The reaction conditions for sequencing were as described previously [12].

\section{Culture collection and nucleotide sequence accession numbers}

C. freundii isolates ARC1 (CCUG42386) and ARC 9 (CCUG42385) have been deposited at the Culture Collection of Gothenburg University, Sweden. GenBank accession numbers were allocated as follows for inf $B$ sequences: ARC1-8, AJ236707-AJ236714; ARC9-15, AJ236716-AJ236722; ASC1-4, AJ236723-AJ236726; and ASC5-7, AJ236728-AJ236730. The accession number AF175203 was allocated for the nucleotide sequence of the integron class 1 of isolate ARC1 containing dhfrXII, orfF and ant $\left(3^{\prime \prime}\right)-I$.

\section{Results}

A county-based monitoring programme during 19931998 disclosed 378 aminoglycoside-resistant Enterobacteriaceae, as reported elsewhere [3]. Forty-one $(11 \%)$ were identified as $C$. freundii and their distribution over time was indicative of an epidemic (Fig. 1). During the first 2 years of surveillance, no aminoglycoside-resistant $C$. freundii (ARC) were found, but four isolates were found in 1995 and 1996, 24 in 1997 and nine in 1998. When the antibiotic-susceptibility profiles of the 41 isolates were compared, one frequent antibiogram (antibiogram type 1) was revealed comprising resistance to streptomycin, gentamicin, netilmicin, tobramycin, tetracycline, sulphonamides and trimethoprim, coupled with susceptibility to amikacin and extended-spectrum cephalosporins. Twenty-eight $(68 \%)$ of the 41 isolates expressed this phenotype; 13 of them were identified during Sept. 1997 (Fig. 1). 


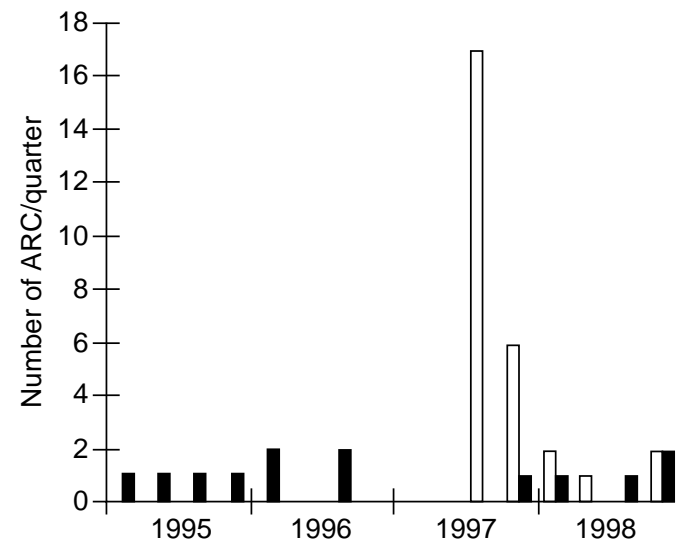

Fig. 1. Quarterly numbers of aminoglycoside-resistant $C$. freundii isolated in the county of Northern Jutland. Only one isolate per patient was allowed. $\square$, C. freundii resistant to streptomycin, gentamicin, netilmicin, tobramycin, tetracycline, trimethoprim and sulphonamides; $\mathbf{\square}$ aminoglycoside-resistant $C$. freundii with other antibiogram types.

These 28 isolates were from patients widely distributed in the county: five of them originated from specimens submitted by general practitioners, 13 from the referral hospital of the county and 10 from five different district hospitals. No association with particular medical specialities or specific hospital departments could be identified (Table 1 and data not shown).

The present study began early in 1998, when 15 of the aminoglycoside-resistant C. freundii from 1997 were chosen for further investigation. The study group comprised the first isolate expressing the antibiogram type 1 , the only aminoglycoside-resistant $C$. freundii with a different antibiogram type collected in 1997 (ARC9) and 13 other initial isolates with antibiogram type 1. Seven isolates without resistance to aminoglycosides (ASC) were included as controls. Four of these were selected from among blood culture isolates collected in 1997 and three from urine specimens. Epidemiological data for the 22 isolates are shown in Table 1.

\section{Typing of C. freundii}

PFGE typing of the 22 isolates revealed eight different profiles (Fig. 2). The 14 resistant isolates of antibiogram type 1 all belonged to the same PFGE type, whereas ARC9, the only gentamicin-resistant isolate susceptible to streptomycin, gave a unique PFGE type. Among the seven aminoglycoside-susceptible organisms, six unique PFGE types were obtained, whereas one susceptible isolate, ASC5, gave the same PFGE type as did resistant isolates of antibiogram type 1 (Fig. 2). Identical conclusions were reached when DNA was restricted with SpeI (Fig. 2) or Xba I (data not shown).

The typing obtained with the RiboPrinter ${ }^{\mathrm{TM}}$ (Fig. 3) correlated well with the PFGE results, dividing the 22 isolates into seven ribogroups; nevertheless, two aminoglycoside-susceptible isolates, ASC3 and ASC7, were discriminated by PFGE (Fig. 2) but not by riboprinting (Fig. 3).

In a previous study on the phylogeny of Enterobacter-

Table 1. C. freundii isolates investigated in the study

\begin{tabular}{lcllc}
\hline Isolate & Month/year & Specimen & Institution & Antibiogram \\
\hline ARC1 & $7 / 97$ & Urine & Rmed1 & 1 \\
ARC2 & $8 / 97$ & Urine & D1sur & 1 \\
ARC3 & $9 / 97$ & Tissue & Rsur1 & 1 \\
ARC4 & $9 / 97$ & Urine & Rmed2 & 1 \\
ARC5 & $9 / 97$ & Urine & GP1 & 1 \\
ARC6 & $9 / 97$ & Urine & D2med1 & 1 \\
ARC7 & $9 / 97$ & Urine & GP2 & 1 \\
ARC8 & $9 / 97$ & Urine & Rmed3 & 1 \\
ARC9 & $10 / 97$ & Sputum & Ricu & 2 \\
ARC10 & $10 / 97$ & Urine & Rmed4 & 1 \\
ARC11 & $10 / 97$ & Wound & GP3 & 1 \\
ARC12 & $10 / 97$ & Urine & Rsur2 & 1 \\
ARC13 & $11 / 97$ & Urine & Rmed5 & 1 \\
ARC14 & $11 / 97$ & Urine & D3reh & 1 \\
ARC15 & $12 / 97$ & Bile & D3sur & 1 \\
ASC1 & $10 / 96$ & Blood & Rsur2 & wt \\
ASC2 & $6 / 97$ & Blood & Rsur3 & wt \\
ASC3 & $9 / 97$ & Blood & Rmed4 & wt \\
ASC4 & $3 / 98$ & Blood** & Rmed1 & wt \\
ASC5 & $9 / 98$ & Urine & D3med & wt \\
ASC6 & $9 / 98$ & Urine & Rmed2 & wt \\
ASC7 & $9 / 98$ & Urine & D2med2 & wt \\
\hline
\end{tabular}

*Referring institution: GP, general practitioner; D, district hospital; R, referral hospital; med, medical department; sur, surgical department; icu, intensive care unit; reh, rehabilitation facility. ${ }^{\dagger}$ Antibiotic susceptibility: wt, wild type susceptible to all aminoglycosides; 1 , resistant to gentamicin, streptomycin, tobramycin, netilmicin, and susceptible to amikacin; 2, resistant to gentamicin, tobramycin, netilmicin, and susceptible to streptomycin and amikacin.

The suspected underlying diseases of the bacteraemic patients were "urinary tract infection, $\S$ cholecystitis, "renal abscess and ${ }^{* *}$ leukaemia. 


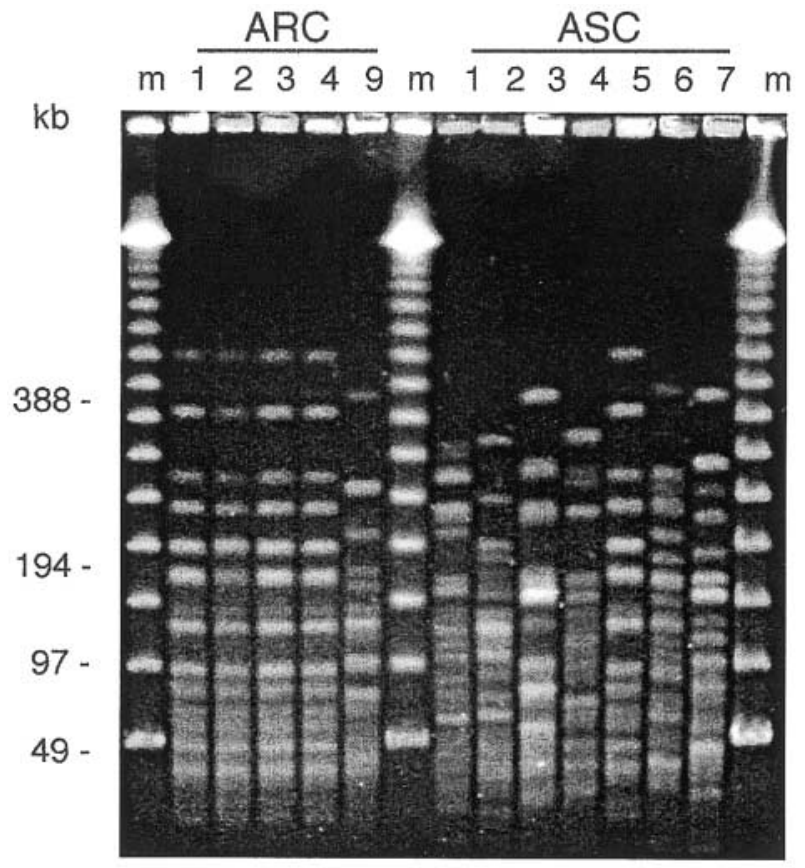

Fig. 2. PFGE DNA patterns of $C$. freundii obtained after restriction with SpeI. Lane $\mathrm{m}, \lambda$ mol. wt standards $(\mathrm{kb})$.

\section{RiboPrint $(R)$ pattern}

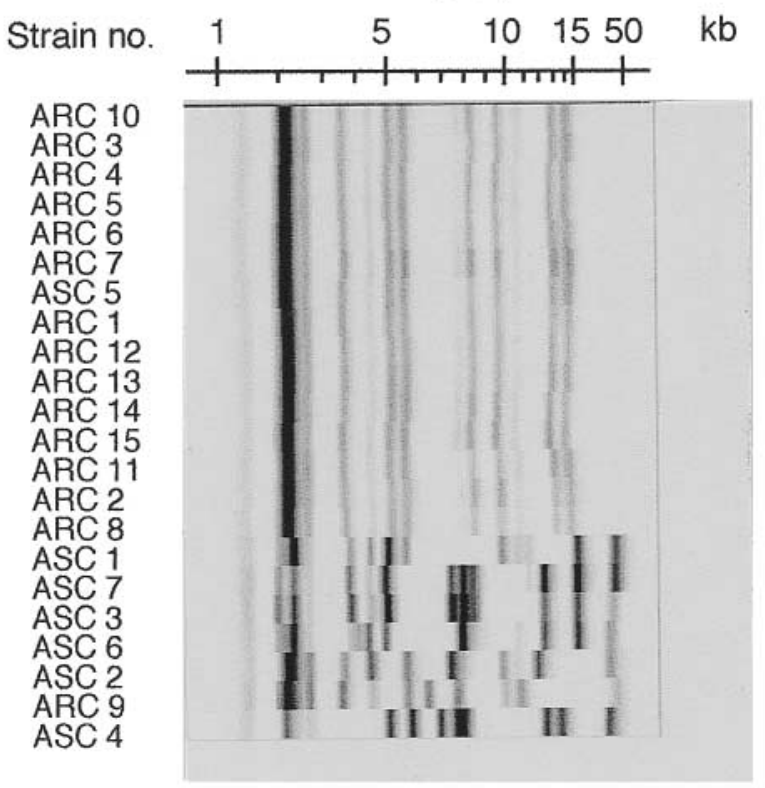

Fig. 3. RiboPrinting results for 22 isolates of $C$. freundii. The mol. wt is indicated at the top. Normalised data are shown for all the organisms, sorted by RiboPrint similarity.

iaceae based on a 450-bp segment of $\inf B$, the nucleotide sequences of two $C$. freundii isolates differed by $1.3 \%$ [4]. To examine whether differences in this gene segment could be of value in the typing of C. freundii, a 417-nucleotide fragment of infB was sequenced and compared for all 22 isolates. No difference was observed with respect to the predicted amino acid sequence, but neutral substitutions were found at the third-base sites of codons in 10 separate positions, giving rise to four sequence groups (Table 2). The distribution of the isolates among sequence groups was in agreement with the results of the other typing methods. Identical sequences were obtained for the susceptible strain ASC5 and the 14 resistant isolates of antibiogram type 1 . Susceptible strains ASC3 and ASC7, which could not be differentiated by the ribotyping, were both placed in sequence group II. The sequence of the resistant isolate ARC9, which differed in antibiogram from the type 1 isolates, was unique.

\section{Aminoglycoside resistance}

To examine the genetic basis of aminoglycoside resistance of ARC1 (as a representative of the epidemic antibiogram type 1 strain) and ARC9, amplification of the genes for six aminoglycoside-modifying enzymes was attempted by PCR. Both isolates were positive for aac(3)-II. Isolate ARC1 also harboured ant(3")-I. These genotypes were in agreement with the phenotypic expression of resistance, with gentamicin, netilmicin and tobramycin being susceptible to inactivation by $\mathrm{AAC}(3)-\mathrm{II}$, and streptomycin to $\mathrm{ANT}\left(3^{\prime \prime}\right)-\mathrm{I}$ (11).

Gene cassettes - mobile elements normally found integrated at a specific site in an integron - may be consecutively inserted in multi-cassette arrays [15]. The $3^{\prime}$ conserved sequence of class I integrons contains a gene (sul1) conferring resistance to sulphonamides. Observation of sulphonamide resistance therefore prompted a search for gene cassettes associated with class I integrons in the multiresistant epidemic strain. PCR-amplification of DNA from isolate ARC1 revealed the presence of a class I integron with an inserted sequence of $\sim 1700 \mathrm{bp}$. Sequencing of the amplification product revealed three open reading frames located in the integron $\left(5^{\prime} \rightarrow 3^{\prime}\right)$. These comprised dhfrXII, conferring resistance to trimethoprim, $\operatorname{orfF}\left(320 \mathrm{bp}\right.$ ) of unknown function [14] and $\operatorname{ant}\left(3^{\prime \prime}\right)-I$ (nucleotide sequence accession no. AF175203).

\section{Discussion}

Attention was drawn to a sudden increase in the prevalence of gentamicin resistance among clinical isolates of $C$. freundii in a Danish county. To examine a possible clonal relationship among these bacteria, 15 isolates were charaterised by three DNA-based typing methods. The discriminatory power varied with the methods, but identical conclusions were reached. Of 15 resistant isolates investigated, 14 were identical as evaluated by typing methods, antibiotic susceptibility and biochemical characteristics. It is generally agreed that isolates indistinguishable by typing schemes, and sharing characteristics that distinguish them from epidemiologically unrelated strains, constitute a cluster, or clone, arising from a common precursor [16]. We 
Table 2. Polymorphic nucleotide sites in a 417-bp segment of the infB gene sequence from 22 C. freundii isolates

\begin{tabular}{|c|c|c|c|c|c|c|c|c|c|c|}
\hline \multirow[b]{2}{*}{ Sequence group } & \multicolumn{10}{|c|}{ Nucleotide position } \\
\hline & 1398 & 1410 & 1431 & 1437 & 1467 & 1473 & 1521 & 1587 & 1629 & 1638 \\
\hline I & $\mathrm{T}$ & A & A & C & $\mathrm{G}$ & G & $\mathrm{G}$ & A & $\mathrm{G}$ & C \\
\hline II & - & - & G & - & - & A & - & - & - & - \\
\hline III & A & - & - & - & - & - & - & G & - & - \\
\hline IV & - & $\mathrm{T}$ & G & $\mathrm{T}$ & A & - & A & G & A & $\mathrm{T}$ \\
\hline
\end{tabular}

The nucleotide numbering is with reference to E. coli [10]. Sequence group I encompasses all aminoglycoside-resistant $C$. freundii of antibiogram type 1, as well as ASC2 and ASC5. The remaining five susceptible isolates constitute sequence groups II (ASC3, 6, 7) and III (ASC1, 4). The nucleotide sequence of group IV is unique to ARC9.

conclude that 14 of 15 resistant isolates investigated belonged to the same epidemic strain and will refer to this as a clone.

Based on antibiotic susceptibility, this clone may account for 28 of 41 aminoglycoside-resistant $C$. freundii isolated in the county from 1993 to 1998. All of these were identified during an 18-month period (Fig. 1). A total of 160 patients in the county had a first-time isolate of $C$. freundii during this 18 -month period, and during this period the resistant clone thus accounted for $18 \%$ of isolates of this genus.

The clone expressed resistance to several antimicrobial agents, and the genes for resistance to trimethoprim, streptomycin and sulphonamides were transcribed from the same promoter in a class I integron. Class I integrons are prevalent among Enterobacteriaceae from various geographic locations, and the presence of a class 1 integron is statistically linked to aminoglycoside resistance [17-19]. dhfrXII was originally described within the same array of gene cassettes in a class I integron from an E. coli isolated in Finland [14]. The deposited sequence from the Finnish isolate (nucleotide sequence accession no. Z21672) is 99.6\% identical with the sequence obtained from ARC1 (data not shown; alignment based on $1087 \mathrm{bp}$ ).

The genes conferring resistance to gentamicin (aac(3)-II) and tetracycline may have been acquired together with the integron; an almost identical integron described previously was borne on a Tn2l-like transposon, and resistance to chloramphenicol and ampicillin was co-transferred together with the integron-determined resistance [14].

One isolate without acquired resistance-ASC5, isolated 12 months after the peak incidence of the epidemic strain-was indistinguishable from the epidemic strain by all typing methods; it may be related to the epidemic strain before acquisition of resistance genes, or it may represent the strain after these genes had been lost.

The source of the outbreak strain remains unresolved. We were unable to trace any sign of cross-infection among patients, and although the majority of repre- sentatives were isolated from hospitalised patients, an origin in the community seems more likely. A cluster of antibiotic-resistant $C$. freundii isolates originating in a community has not been described previously. Nevertheless, some parallels may be drawn with an epidemic of multiresistant $E$. coli infection recorded in London in 1986-1988 [20,21] and also acquired in the community. Most isolates of this latter clone were identified during a 4-month period, after which it gradually disappeared.

The multiresistance of the epidemic strain offers selective advantages in the presence of antibiotics, and sulphonamides and trimethoprim are widely employed in the treatment of cystitis in Denmark. $C$. freundii occurs not only in the animal intestine but also in water, soil and food [22]. Due to this ubiquitous presence in the environment, selection of the epidemic strain may have occurred before patient acquisition of the bacterium.

Attention was drawn to gentamicin resistance because of the continuing role of this antibiotic in the treatment of serious infection in some countries. Spread of aminoglycoside resistance among enteric bacilli has occurred as a consequence of high consumption of these antibiotics in some hospital departments [23, 24]. In the present study, resistance to gentamicin may have served only as a marker of the epidemic strain.

The study was supported by grants from speciallæge Heinrich Kopp's Legat and Nordjyllands Lægekredsforenings Forskningsfond. H.U.S-P. received financial support from the Familien Hede Nielsens Fund and the Biotechnology Programme of the Danish Natural Science Research Council (28807-9502036, 9602401).

\section{References}

1. Harbarth S, Rohner P, Safran E, Garbino J, Auckenthaler R, Pittet D. Resistance to amikacin and gentamicin among gramnegative bloodstream isolates in a university hospital between 1989 and 1994. Clin Microbiol Infect 1998; 4: 199-204.

2. Schønheyder HC, Højbjerg T. The impact of the first notification of positive blood cultures on antibiotic therapy. APMIS 1995; 103: 37-44.

3. Schønheyder HC, Sanden AK, Sørensen HT. The prevalence of gentamicin resistance among clinical isolates of enterobacteria in a Danish region. APMIS 2000; 108: 145-152.

4. Hedegaard J, Steffensen SA, Nørskov-Lauritsen N, Mortensen KK, Sperling-Petersen HU. Identification of Enterobacteriaceae 
by partial sequencing of the gene encoding translation initiation factor 2. Int J Syst Bacteriol 1999; 49: 1531-1538.

5. Pfaller MA, Jones RN, Marshall SA et al. Inducible Amp C $\beta$ lactamase producing gram-negative bacilli from blood stream infections: frequency, antimicrobial susceptibility, and molecular epidemiology in a national surveillance program (SCOPE). Diagn Microbiol Infect Dis 1997; 28: 211-219.

6. Papasian CJ, Kinney J, Coffman S, Hollis RJ, Pfaller MA. Transmission of Citrobacter koseri from mother to infant documented by ribotyping and pulsed-field gel electrophoresis. Diagn Microbiol Infect Dis 1996; 26: 63-67.

7. El Harrif-Heraud Z, Arpin C, Benliman S, Quentin C. Molecular epidemiology of a nosocomial outbreak due to SHV-4-producing strains of Citrobacter diversus. $J$ Clin Microbiol 1997; 35: 2561-2567.

8. Janda JM, Abbott SL, Cheung WKW, Hanson DF. Biochemical identification of Citrobacteria in the clinical laboratory. J Clin Microbiol 1994; 32: 1850-1854.

9. Foodborne and Diarrheal Disease Branch, Division of Bacterial and Mycotic Diseases, National Center for Infectious Diseases, Centers for Disease Control and Prevention. Standardized molecular subtyping of Escherichia coli $\mathrm{O} 157: \mathrm{H} 7$ by pulsedfield gel electrophoresis: a training manual. Atlanta, Centers for Disease Control and Prevention. 1996.

10. Blattner FR, Plunkett G, Bloch CA et al. The complete genome sequence of Escherichia coli K-12. Science 1997; 277: 1453-1474.

11. Shaw KJ, Rather PN, Hare RS, Miller GH. Molecular genetics of aminoglycoside resistance genes and familial relationships of the aminoglycoside-modifying enzymes. Microbiol Rev 1993; 57: $138-163$

12. Sandvang D, Aarestrup FM, Jensen LB. Characterisation of integrons and antibiotic resistance genes in Danish multiresistant Salmonella enterica DT104. FEMS Microbiol Lett 1998; 160: 37-41.

13. Kazama H, Kizu K, Iwasaki M, Hamashima H, Sasatsu M, Arai $\mathrm{T}$. Isolation and structure of a new integron that includes a streptomycin resistance gene from the $\mathrm{R}$ plasmid of Pseudomonas aeruginosa. FEMS Microbiol Lett 1995; 134: 137-141.
14. Heikkilä E, Skurnik M, Sundström L, Huovinen P. A novel dihydrofolate reductase cassette inserted in an integron borne on a Tn21-like element. Antimicrob Agents Chemother 1993; 37: $1297-1304$.

15. Recchia GD, Hall RM. Gene cassettes: a new class of mobile element. Microbiology 1995; 141: 3015-3027.

16. Power EGM. RAPD typing in microbiology. A technical review. J Hosp Infect 1996; 34: 247-265.

17. Martinez-Freijo P, Fluit AC, Schmitz F-J, Grek VSC, Verhoef $\mathrm{J}$, Jones ME. Class I integrons in gram-negative isolates from different European hospitals and association with decreased susceptibility to multiple antibiotic compounds. J Antimicrob Chemother 1998; 42: 689-696.

18. Martinez-Freijo P, Fluit AC, Schmitz F-J, Verhoef J, Jones ME Many class I integrons comprise distinct stable structures occurring in different species of Enterobacteriaceae isolated from widespread geographic regions in Europe. Antimicrob Agents Chemother 1999; 43: 686-689.

19. Sallen B, Rajoharison A, Desvarenne S, Mabilat C. Molecular epidemiology of integron-associated antibiotic resistance genes in clinical isolates of enterobacteriaceae. Microb Drug Resist 1995; 1: 195-202.

20. O'Neill PM, Talboys CA, Roberts AP, Azadian BS. The rise and fall of Escherichia coli $\mathrm{O} 15$ in a London teaching hospital. J Med Microbiol 1990; 33: 23-27.

21. Phillips I, Eykyn S, King A et al. Epidemic multiresistant Escherichia coli infection in West Lambeth Health District. Lancet 1988; 1: 1038-1041.

22. Sakazaki R. Genus IV. Citrobacter Werkman and Gillen 1932 , $173^{\mathrm{AL}}$. In: Krieg NR, Holt JG (eds) Bergey's Manual of systematic bacteriology, vol 1. Baltimore, Williams \& Wilkins. 1984: 458-461.

23. Aronsson B, Ericsson M, Herin P, Rylander M. Gentamicinresistant Klebsiella spp. and Escherichia coli in a neonatal intensive care unit. Scand J Infect Dis 1991; 23: 195-199.

24. Johansen KS, Storgaard M, Carstensen N, Frank U, Daschner F. An international study of the occurrence of multiresistant bacteria and aminoglycoside consumption patterns. Infection 1998; 16: 313-322. 